Andreas Fisahn / Regina Viotto

\title{
Privatisierung und Public-Private-Partnership
}

\section{Formen der Aufgabenwahrnehmung und der Privatisierung}

Mit dem Zusammenbruch der US-Immobilienmärkte und den folgenden Bankenkrisen gerät der Marktfundamentalismus auch bei seinen Vordenkern in Misskredit. Anhaltend ist dennoch der Trend zur umfassenden Privatisierung von öffentlich erbrachten Leistungen und Gütern. Allerdings sind hier inzwischen - z.B. im Bereich der Müllabfuhr - Einzelfälle einer Rekommunalisierung auszumachen, die diese Entwicklung aber noch nicht umkehren. Zunehmend stößt - wie die folgenden Überlegungen zeigen - die Privatisierung an funktionale und verfassungsrechtliche Grenzen.

Es ist weitgehend anerkannt, dass es öffentliche Aufgaben gibt, die zunächst durch ein öffentliches Interesse gekennzeichnet werden können, im allgemeinen Interesse liegen oder dem Allgemeinwohl dienen. Das BVerfG hat das so formuliert: mit öffentlichen Aufgaben »sind die Aufgaben gemeint, an deren Erfüllung ein gesteigertes Interesse der Gemeinschaft besteht, die aber so geartet sind, dass sie weder im Wege privater Initiative wirksam wahrgenommen werden können noch zu den im engeren Sinn staatlichen Aufgaben zählen, die der Staat selbst durch seine Behörden wahrnehmen muss.«(BVerfGE 38, 281/ 299) Öffentliche Aufgaben werden also von Aufgaben, die durch private Initiative erfüllt werden können und im engeren Sinne staatlichen Aufgaben abgegrenzt. Alle drei verbindet, dass an ihnen ein öffentliches Interesse oder ein gesteigertes Interesse der Gemeinschaft besteht. Und selbstverständlich besteht ein gesteigertes Interesse der Gemeinschaft etwa an der Versorgung mit Lebensmitteln, aber dies muss - jedenfalls unter gegenwärtigen Bedingungen einer ausreichenden Versorgung - nicht öffentlich organisiert werden. Das Beispiel weist schon darauf hin, unter welchen Bedingungen die öffentliche Aufgabe auch öffentlich organisiert werden muss: offenbar unter Bedingungen, unter denen eine gleichmäßige Versorgung auf dem Niveau eines kulturellen Minimums privat nicht erbracht werden kann, wobei die gleichmäßige Versorgung auf die Berücksichtigung von Gleichheitsaspekten, die sich aus dem Gleichheitsgebot des Art. 3 GG und dem Sozialstaatsprinzip ergeben, abstellt.

Die staatliche Aufgabe wird vom BVerfG eher tautologisch bestimmt, als öffentliche Aufgabe, derer sich der Staat annimmt. »Die Veranstaltung von Rundfunksendungen ist nach der deutschen Rechtsentwicklung eine öffentliche Aufgabe. Wenn sich der Staat mit dieser Aufgabe in irgendeiner Form befasst (auch dann, wenn er sich privatrechtlicher Formen bedient), wird sie zu einer ,staatlichen Aufgabe' im Sinne von Art. 30 GG.« (BVerfGE 12, 205/ 205) Die höchstrichterliche Definition der »öffentlichen Aufgabe « impliziert, dass es Aufgaben gibt, die der Staat selbst wahrnehmen muss, die also weder materiell, noch funktionell und teilweise auch nicht formell privatisiert werden darf.

Dabei bedeutet materielle Privatisierung, dass die Erledigung der Aufgaben nach ihrer Rechtsform und nach ihrer Erledigungsform Privaten überantwortet wird. Funktio- 
nelle Privatisierung bedeutet, dass der Staat sich Privater zur Erfüllung der Aufgabe bedient, aber Träger und verantwortlich für die Aufgabe bleibt. Hierunter fallen vor allem die teilweise Aufgabenübertragung an Private, meist in Form der Beleihung und die PPP. Formelle Privatisierung heißt, dass der Staat die Aufgabe einer privatrechtlich organisierten Einheit überträgt, aber deren Eigentümer bleibt.

\section{Unmittelbare verfassungsrechtliche Zuordnungen}

\section{Wirtschaftspolitische Neutralität des $G G$}

Die staatliche Aufgabenverantwortung ist zunächst vor dem Hintergrund der wirtschaftspolitischen Neutralität des Grundgesetzes, die das BVerfG mehrfach betont hat, zu betrachten. Wirtschaftspolitische Neutralität bedeutet, dass es dem politischen Prozess, den parlamentarischen Mehrheiten überlassen bleibt, die Wirtschaftsform festzulegen. Das BVerfG führte im Urteil zur Verfassungskonformität der Investitionshilfe aus: »Das Grundgesetz garantiert weder die wirtschaftspolitische Neutralität der Regierungs- und Gesetzgebungsgewalt noch eine nur mit marktkonformen Mitteln zu steuernde , soziale Marktwirtschaft‘. Die, wirtschaftspolitische Neutralität' des Grundgesetzes besteht lediglich darin, dass sich der Verfassungsgeber nicht ausdrücklich für ein bestimmtes Wirtschaftssystem entschieden hat. Dies ermöglicht dem Gesetzgeber die ihm jeweils sachgemäß erscheinende Wirtschaftspolitik zu verfolgen, sofern er dabei das Grundgesetz beachtet. Die gegenwärtige Wirtschafts- und Sozialordnung ist zwar eine nach dem Grundgesetz mögliche Ordnung, keineswegs aber die allein mögliche. Sie beruht auf einer vom Willen des Gesetzgebers getragenen wirtschafts- und sozialpolitischen Entscheidung, die durch eine andere Entscheidung ersetzt oder durchbrochen werden kann « (BVerfGE 4, 7). Daraus folgt, dass dem Gesetzgeber verfassungsrechtlich ein weiter Ermessensspielraum eingeräumt wird, die wirtschaftspolitische Verfasstheit der Bundesrepublik zu bestimmen. Das Grundgesetz schreibt genauso wenig eine marktwirtschaftliche Organisation wie Formen einer solidarischen Ökonomie vor, sondern überlässt die Wahl der wirtschaftspolitischen Organisation dem Gesetzgeber. Ein weites Ermessen hat das BVerfG mehrfach für die Wahl der Form der Organisation staatlicher Aufgabenwahrnehmung eingeräumt: Es ist danach »Sache des gesetzgeberischen Ermessens, zu entscheiden, welche dieser Aufgaben der Staat nicht durch seine Behörden, sondern durch eigens gegründete öffentlich-rechtliche Anstalten oder Körperschaften erfüllt. Das Bundesverfassungsgericht kann hierbei nur nachprüfen, ob der Gesetzgeber die Grenzen seines Ermessens beachtet hat.« (BVerfGE 10, 89/ 102) Dies gilt zunächst für die Wahl der öffentlichen Organisation der Aufgabenwahrnehmung, ist aber unter dem Gesichtspunkt der wirtschaftspolitischen Neutralität der Verfassung auch für die Frage anzunehmen, ob Aufgaben privatwirtschaftlich oder öffentlich ausgeführt werden.

Mit Blick auf die private oder öffentliche Organisation der Aufgabenerfüllung hat das BVerfG dem Gesetzgeber deshalb ebenfalls ein Ermessen eingeräumt, aber gleichzeitig verlangt, dass die Qualität der Erfüllung öffentlicher Aufgaben gesichert bleiben müsse: »Wie der Staat öffentliche Aufgaben erledigen lassen will, ist im allgemeinen 
Sache seines freien Ermessens, freilich bis zu einem gewissen Grade auch von Eigenart und Gewicht der einzelnen Aufgabe abhängig. Es besteht hier eine breite Skala von Möglichkeiten, die vom freien Beruf mit öffentlich-rechtlichen Auflagen bis zu Berufen reicht, die gänzlich in die unmittelbare Staatsorganisation einbezogen sind, also ,öffentlichen Dienst“ im eigentlichen Sinne darstellen.«(BVerfGE 17, 371/377)

\section{Verfassungsrechtliche Normierung der Form der Aufgabenerfüllung}

Das grundsätzliche Ermessen des Bundesgesetzgebers bei Entscheidungen, wie öffentliche Aufgaben zu organisieren sind, wird allerdings für einige Bereiche durch das Grundgesetz selbst begrenzt. Dazu gehört etwa die Organisation der Telekommunikation und der Postdienste, die nämlich seit der Verfassungsänderung von 1993 in groBen Teilen privatwirtschaftlich organisiert werden sollen. Art 87 f Abs. 2 GG fordert für die Organisation der Telekommunikation, dass die Dienstleistungen als privatwirtschaftliche Tätigkeiten durch die aus dem Sondervermögen Deutsche Bundespost hervorgegangenen Unternehmen und durch andere private Anbieter erbracht werden. Dagegen sollen Hoheitsaufgaben im Bereich des Postwesens und der Telekommunikation in bundeseigener Verwaltung ausgeführt werden.

Für die Post schreibt Art. 143 b Abs. 1 GG zwingend eine formelle Privatisierung vor, da das Sondervermögen Deutsche Bundespost nach Maßgabe eines Bundesgesetzes in ein Unternehmen privater Rechtsform umgewandelt werden soll, was bekanntlich geschehen ist. Das heiße allerdings nicht, so das BVerfG ausdrücklich, dass die Erbringung der Postdienstleistungen uneingeschränkt auf das Wettbewerbsprinzip festgelegt werde. »Privatwirtschaftlichkeit«, führt das Gericht aus, »verweist zunächst auf den Grundsatz der Wirtschaftlichkeit und auf eine am Gewinnprinzip orientierte Betätigung; sie kann beispielsweise auch gegeben sein, wenn ein privatwirtschaftliches Unternehmen ohne Wettbewerber handelt, wie dies gegenwärtig in einem erheblichen Teil der Universaldienstleistungen der Fall ist. Eine Auslegung des Art. $87 \mathrm{f}$ Abs. 2 GG, die ausnahmslos auf die Schaffung von Wettbewerb hinausläuft, wird vom Grundgesetz unter keinem rechtlichen Gesichtspunkt gestützt.« (BVerfG, 1 BvR 1712/ 01 vom 7.10.2003, Absatz-Nr.95)

Auch im Bereich der Bahn schreibt das Grundgesetz nicht die Schaffung eines wettbewerbsorientierten Systems vor, vielmehr wird - im Gegenteil - sowohl für die Bahn wie für Post und Telekommunikation der Staat auf eine am Allgemeinwohl orientierte Organisation und Erbringung der jeweiligen Dienstleistungen verpflichtet, was noch zu diskutieren ist.

Umgekehrt schreibt das Grundgesetz für andere Bereiche zwingend vor, dass sie staatlich organisiert werden. So bestimmt Art. 90 Abs. 1 GG in der klassisch kurz und bündigen Form: Der Bund ist Eigentümer der bisherigen Reichsautobahnen und Reichsstraßen, die also nicht privatisiert werden dürfen. Oder Art. 87 a GG: Der Bund stellt Streitkräfte zur Verteidigung auf, die natürlich nicht privatrechtlich organisiert werden können. Das gleiche gilt für die Behörden nach Art. 87 Abs. 1 GG, wonach Bundesgrenzschutzbehörden, Zentralstellen für das polizeiliche Auskunfts- und Nachrichtenwesen, für die Kriminalpolizei und zur Sammlung von Unterlagen für Zwecke 
des Verfassungsschutzes eingerichtet werden können. Ein gesetzgeberisches Schelmenstück, das zu entsprechenden Diskussionen geführt hat, ist Art. 87 d Abs. 1 GG, der bestimmt: »Die Luftverkehrsverwaltung wird in bundeseigener Verwaltung geführt. Über die öffentlich-rechtliche oder privat-rechtliche Organisationsform wird durch Bundesgesetz entschieden. « Nachdem der Bundespräsident im April 2006 die Änderungen des Luftverkehrsgesetzes, mit der die Luftsicherung privatisiert werden sollte, nicht unterzeichnet hat, ist die Mehrheit der Juristen der Überzeugung, dass die Luftverkehrsverwaltung nicht unter Aufgabe der Kontrollrechte des Bundes und seiner Mehrheitsbeteiligung privatisiert werden darf.

\section{Gewaltmonopol und staatliche Form der Aufgabenerfüllung}

Die hier ausgewählten Beispiele erhellen, dass insbesondere die Organisation der »inneren und äußeren Sicherheit«, d.h. von Militär, Polizei und Geheimdiensten eine öffentliche Aufgabe ist, die staatlich organisiert werden muss, in diesem Fall durch staatliche Einrichtungen wahrgenommen werden muss. Hier handelt es sich um eine notwendige staatliche Aufgabe, die auch nicht formell privatisiert, also privatrechtlich organisiert werden darf. Das Gewaltmonopol bestimmt die notwendigen Aufgaben des Staates, weil der Staat über dieses definiert wird, also nur dort ein Staat existiert, wo es eine Staatsgewalt mit halbwegs effektivem Gewaltmonopol gibt. Vom Grundgesetz wird es vorausgesetzt, was in der Bindung der staatlichen Gewalt an die Verfassung in Art. 1 Abs. 3 i.V.m. 20 Abs. 2, 3 GG zum Ausdruck kommt. Mit einer Privatisierung, die den Bereich des Gewaltmonopols betrifft, würde gleichsam das »Wesen« des Staates tangiert oder »Staatlichkeit« selbst ausgehöhlt, was mit der verfassungsmäßigen Ordnung nicht zu vereinbaren ist. (Ehlers, Aushöhlung der Staatlichkeit durch die Privatisierung von Staatsaufgaben, S. 108) Das Gewaltmonopol ist an dieser Stelle, wie man den zitierten Vorschriften des Grundgesetzes entnehmen kann, eng mit dem Rechtsstaatsprinzip verwoben. Nur wo die Gewalt rechtlich gebunden ist und am Maßstab des Rechts kontrolliert und beschränkt wird, rechtfertigt sich deren Monopolisierung beim Staat. Im Unterschied zu Privaten ist die staatliche Gewalt unmittelbar an die Grundrechte, die Verfassung und die Gesetze gebunden. Dieser Unterschied der verfassungsrechtlichen Bindung macht ebenso wie das Gewaltmonopol den Kernbereich der rechtsstaatlichen Ordnung im modernen Verfassungsstaat aus und setzt gleichzeitig die Grenzen für eine Privatisierung dieses Kernbereichs staatlicher Gewalt.

Dann folgt, dass beispielsweise Polizei und Strafvollzug (Gusy, JZ 2006, 651) grundsätzlich nicht privatisiert werden dürfen. Im Bereich der Polizei besteht dabei vor allem die Gefahr einer schleichenden Privatisierung durch die Zunahme privater Sicherheitsdienste, die vereinzelt mit beschränkten Kompetenzen das Gewaltmonopol des Staates nicht tangieren, dieses aber zurückdrängen und aushöhlen können, wenn der Eindruck entsteht, dass insbesondere die alltägliche Sicherheit vom Staat nicht mehr zu gewährleisten ist und privat organisiert werden muss (Steegmann, in: Gusy Privatisierung von Staatsaufgaben, 237/248). Verfassungsrechtlich bedenklich ist es in diesem Sinne auch, wenn der Staat einzelne Lebensbereiche oder Situationen vollständig privaten Sicherheitsfirmen überlässt. 


\section{Wahrnehmung hoheitsrechtlicher Befugnisse durch Private - funktionelle Privati- sierung}

\section{Funktionsvorbehalt und funktionelle Privatisierung}

Die Übertragung hoheitsrechtlicher Befugnisse ist Maßstab zur Beurteilung der Privatisierung in den Formen der formellen und der funktionalen Privatisierung, wobei insbesondere letztere verfassungsrechtliche Probleme aufwirft, weil hier Privaten, sog. Beliehenen, die Wahrnehmung hoheitsrechtlicher Aufgaben übertragen wird, oder im Falle der PPP Private an öffentlich-rechtlichen Einrichtungen in der Form beteiligt werden, dass sie faktisch an der Wahrnehmung hoheitsrechtlicher Aufgaben mitwirken und auf diese Einfluss nehmen können.

Nahe am Gewaltmonopol liegend, aber dennoch über dessen normative Implikationen hinausgehend ist die Bestimmung in Art. 33 Abs. 4 GG, wonach die Ausübung hoheitsrechtlicher Befugnisse als ständige Aufgabe in der Regel Angehörigen des öffentlichen Dienstes zu übertragen ist, die in einem öffentlich-rechtlichen Dienst- und Treueverhältnis stehen. Der Begriff der hoheitsrechtlichen Befugnisse wird zwar immer wieder mal verwendet, ist aber in seiner positiven Substanz umstritten. Einigkeit besteht, dass hoheitliche Befugnisse sich nicht auf die sog. Eingriffsverwaltung beschränken, sondern ebenso im Bereich der Leistungsverwaltung anzutreffen sind, schon weil aufgrund dieser Unterscheidung keine klare Trennlinie zu ziehen ist. (Kunig, in: Münch/Kunig, Art. 33 GG, Rn. 47 ff.) Hoheitsrechtliche Befugnisse unterscheiden sich von privatrechtlichen durch die Weite des Bereichs, in dem eine Kompetenz besteht, einseitig verbindliche Entscheidungen zu treffen. Das geschieht sowohl im Bereich der allgemeinen Dienstleistungen und der Infrastrukturleistungen wie im Bereich des Eingriffs in Grundrechte.

Die genauere Bestimmung der hoheitsrechtlichen Befugnisse konnte oftmals dahinstehen, weil Art. 33 Abs. 4 GG ein Regel-Ausnahme-Verhältnis normiert. Die Rechtsprechung konnte in einigen Fällen die Privatisierung auch von Bereichen mit hoheitsrechtlichen Befugnissen für verfassungskonform erklären, weil die hoheitsrechtlichen Befugnisse nur »in der Regel « von Beamten ausgeübt werden sollen, woraus gefolgert wird, dass Ausnahmen auch in dem Sinne zulässig sind, dass hoheitliche Aufgaben Privaten übertragen werden. (BVerwGE 7 BN 2/05, v. 29.9.2005, Absatz-Nr.4)

Um dem Regel-Ausnahme-Verhältnis Rechnung zu tragen, ist es verfassungsrechtlich geboten, dass die Übertragung hoheitsrechtlicher Aufgaben auf Private durch Gesetz oder aufgrund eines Gesetzes geschieht (BVerfG NJW 1987, 2501/ 2502, BVerwGE 98, 280/298), wobei die funktionale Privatisierung durch sachliche Gründe gerechtfertigt sein muss, so dass die Kontinuität hoheitlicher Funktionen des Staates gewahrt bleibt. (BVerfGE 88, 103/ 114; BVerwGE 7 BN 2/05, v. 29.9.2005, AbsatzNr.4) Dabei ist zu beachten, dass ausschließlich fiskalische Erwägungen keinen solchen sachlichen Grund darstellen. Schließlich ist dabei darauf zu achten, dass das Regel-Ausnahme-Verhältnis gewahrt wird. (BVerfGE 9, 268/284) Daraus lässt sich einerseits schließen, dass die Beleihung von Privaten mit hoheitlichen Befugnissen grundsätzlich zulässig ist (BVerwGE 57, 55/ 60). Andererseits ist aber bei jeder Ent- 
scheidung im Einzelfall geboten zu überprüfen, inwieweit mit der Übertragung von hoheitlichen Befugnissen das Regel-Ausnahme-Verhältnis gewahrt bleibt. Das heißt nicht, dass nur eine quantifizierende Betrachtung erfolgen muss. So eine Betrachtung würde beispielsweise bei der funktionalen Privatisierung der Kommunalverwaltungen nach etwa britischem Vorbild zu dem Ergebnis führen, dass das Regel-Ausnahme Verhältnis des Art. 33 Abs. 4 GG verletzt ist. Es bedeutet auch, dass im Einzelfall zu prüfen ist, ob durch die Übertragung der hoheitsrechtlichen Befugnisse der Kernbereich staatlicher Aufgaben im Sinne der Gewährleistung von Sicherheit betroffen ist. Je enger die funktionale Privatisierung an diesen Kernbereich heranrückt und je mehr wertende - im Unterschied zu technischen - Entscheidungen zu treffen sind, um so eher wird man ebenfalls zu einem Verstoß gegen das Regel-Ausnahme-Verhältnis kommen müssen. Dies hat das BVerwG beispielsweise in dem Fall der Übertragung hoheitsrechtlicher Kontroll- und Entscheidungsbefugnisse bei besonders überwachungsbedürftigen Abfällen (BVerwG v. 29.9.2005, a.a.O.) ungeprüft gelassen und so im Ergebnis die Bedeutung des Art. 33 Abs. 4 GG verkannt.

\section{Gesetzesvorbehalt und Privatisierung}

Die Übertragung hoheitlicher Befugnisse auf Private in Form der Beleihung oder der PPP bedarf - wie gesehen - nach gefestigter Rechtsprechung der gesetzlichen Grundlage. Dies ist zu begründen mit dem dem Demokratieprinzip entspringenden Gesetzesvorbehalt, der ein förmliches Gesetz für alle Regelungen der im Kompetenzbereich des Staates liegenden Angelegenheiten, die von grundsätzlicher Bedeutung für die Allgemeinheit sind, verlangt. Er verpflichtet den Gesetzgeber, «in grundlegenden normativen Bereichen, zumal im Bereich der Grundrechtsausübung, soweit diese staatlicher Regelung zugänglich ist, alle wesentlichen Entscheidungen selbst zu treffen « (BVerfGE 49, 89 /126; 61, 260 /275; 88, 103 /116; vgl. Ossenbühl, Vorrang und Vorbehalt des Gesetzes, in: Isensee/Kirchhof, Handbuch des Staatsrechts, Bd. III, 2. Aufl. 1996, § 62 Rdnr. 35 ff.; Schulze-Fielitz in: H. Dreier, Grundgesetz-Kommentar, Bd. 2, 1998, Art. 20 / Rechtsstaat Rdnr. 104). Der demokratische Gesetzesvorbehalt soll sicherstellen, dass der Gesetzgeber sich nicht durch offene oder versteckte Delegation beispielsweise in Form von Generalklauseln seiner Gesetzgebungsaufgabe und damit seiner demokratischen Verantwortung gegenüber dem Souverän entzieht und hoheitliche Funktionen jenseits demokratischer Verantwortung wahrgenommen werden. (Ossenbühl, a.a.O., Rn. 42)

Daraus folgert der Staatsgerichtshof Bremen: »Der demokratische Gesetzesvorbehalt verlangt daher nicht nur gesetzgeberisches Selbstentscheiden in grundlegenden Angelegenheiten des Gemeinwesens, sondern darüber hinaus auch eine hinlängliche Regelungsdichte des Gesetzes, um zu vermeiden, dass der Exekutive beim Vollzug des Gesetzes so große Entscheidungsspielräume bleiben, dass in Wirklichkeit sie die dem Gesetzgeber vorbehaltene politische Gestaltungsaufgabe wahrnimmt.« (StGH Bremen St 1/01 v. 15.1.2002, S. 16 f.) Diese Regelungsdichte ist beim Akt der Beleihung zu beachten. Das Gesetz, das hoheitliche Befugnisse überträgt oder auf dessen Grundlage diese übertragen werden, muss also so konkret gefasst sein, dass die Wahr- 
nehmung der hoheitsrechtlichen Aufgaben den Erfordernissen einer demokratischen Programmierung der Exekutive und der demokratischen Verantwortlichkeit genügen. Das heißt, es ist gesetzlich möglichst konkret zu bestimmen, welche Entscheidungsbefugnisse - zukünftig von Privaten - wahrgenommen werden und an welchen normativen Maßstäben sich die Entscheidungen zu orientieren haben. Bei den Anforderungen an die gesetzliche Konkretisierung wird man auf die Art oder Weite der Entscheidungsbefugnisse, ihren wertenden, politischen oder rein technischen Charakter abstellen müssen.

Schließlich umfasst der demokratische Gesetzesvorbehalt bei der Übertragung von Hoheitsrechten die organisationelle Einbindung und Kontrolle der Institutionen, die Hoheitsrechte wahrnehmen oder an Entscheidungen mitwirken können. Wenn es sich weiter um eine Staatsfunktion handelt, für die der Staat letztlich die Verantwortung trägt, wird sie - jedenfalls theoretisch - der Volksvertretung zugerechnet, die sich gegenüber dem demokratischen Souverän zu verantworten hat. Dann ist es unabdingbar, dass mit dem Akt der Übertragung für den Gesetzgeber erkennbar ist, in welcher organisationellen Form diese Zurechnung erfolgen könnte oder wird. Über die Organisations- und Verfahrensregelungen werden schließlich letztlich auch die Grundrechte der Bürger geschützt. (BVerfGE 53, 30/ 59 f) Diese Bedeutung der prozeduralen Regelungen für die staatlichen Schutzpflichten und Verantwortungszusammenhänge hat beispielsweise der VerfGH Berlin in seinem Urteil zur BWB (VerfGH Berlin, DVBl. 2000, S. 54) verkannt.

\section{Funktionelle Privatisierung und Demokratieprinzip}

Die Mit-Wahrnehmung hoheitlicher Befugnisse durch Private ist schließlich am Demokratieprinzip des Art. 20 Abs. 2 GG zu messen. »Alle Staatsgewalt geht vom Volke aus «, wird von der h.M. und Rechtsprechung als Gebot der demokratischen Legitimation der Ausübung von Staatsgewalt verstanden. Da die Wahrnehmung hoheitlicher Befugnisse regelmäßig auch als Ausübung von Staatsgewalt zu verstehen ist, sind jenseits der Kritik an der eingeschränkten Auslegung des Demokratieprinzips als Legitimation von Herrschaft (vgl. Fisahn, Demokratie und Öffentlichkeitsbeteiligung, S. 216 ff.) - mindestens dessen Voraussetzungen im Falle einer funktionellen Privatisierung zu beachten. Neben der gesetzlichen Programmierung der Exekutive verlangt das Demokratieprinzip die personelle und sachlich-inhaltliche Legitimation der Staatsgewalt. Organe und Amtswalter bedürfen danach »zur Ausübung von Staatsgewalt einer Legitimation, die - als eine demokratische - auf die Gesamtheit der Staatsbürger, das Volk, zurückgeht, jedoch regelmäßig nicht durch unmittelbare Volkswahl erfolgen muss. In diesem Bereich ist die Ausübung von Staatsgewalt demokratisch legitimiert, wenn sich die Bestellung der Amtsträger - personelle Legitimation vermittelnd - auf das Staatsvolk zurückführen lässt und das Handeln der Amtsträger selbst eine ausreichende sachlich-inhaltliche Legitimation erfährt; dies setzt voraus, dass die Amtsträger im Auftrag und nach Weisung der Regierung ... handeln können und die Regierung damit in die Lage versetzen, die Sachverantwortung gegenüber Volk und Parlament zu übernehmen.« (BVerfGE 93, 37/ 67) Uneingeschränkte personelle Legitimation be- 
sitzt ein Amtsträger dann, wenn er sein Amt im Wege einer Wahl durch das Volk oder das Parlament oder dadurch erhalten hat, dass er durch einen seinerseits personell legitimierten, unter Verantwortung gegenüber dem Parlament handelnden Amtsträger oder mit dessen Zustimmung bestellt worden ist. Verlangt wird die sog. ununterbrochene Legitimationskette. (BVerfGE 83, 60/73)

Die personelle Legitimationskette verlangt, dass die Amtswalter von einem seinerseits legitimierten Amtsträger eingesetzt werden und die letztliche Legitimation gründet auf der Wahl der Regierung durch das Parlament. Im Falle einer Beteiligung sog. nicht legitimierter Personen verlangt das BVerfG, dass im Entscheidungsverfahren die legitimierten Amtswalter entweder im Gremium in der Mehrheit sind und in ihrer Mehrheit zustimmen oder das Letztentscheidungsrecht haben: Abschließende Entscheidungen können nur »unter der Voraussetzung hingenommen werden, dass die Mehrheit ihrer Mitglieder uneingeschränkt personell demokratisch legitimiert ist und die Entscheidung darüber hinaus von einer Mehrheit der so legitimierten Mitglieder getragen wird.« Gefordert wird das Prinzip der sogenannten doppelten Mehrheit. Allerdings könne der Gesetzgeber den gemischten Gremien anhaftenden Mangel demokratischer Legitimation durch das Letztentscheidungsrecht einer in parlamentarischer Verantwortung stehenden oder dem Weisungsrecht eines parlamentarisch verantwortlichen Amtsträgers unterliegenden Stelle ausgleichen. (BVerfGE 83, 60/ 71; Böckenförde, Demokratie als Verfassungsprinzip, in: Isensee/Kirchhof, Handbuch des Staatsrechts, Bd. I, 1987, § 22, S. 899, Fußnote 25) Dann ist zu folgern, dass PPP Vereinbarungen in Fällen, in denen hoheitliche Befugnisse übertragen und wahrgenommen werden, nur dann den Erfordernissen der ununterbrochenen Legitimationskette genügen, wenn das Prinzip der doppelten Mehrheit in den Gremien für Entscheidungen mit hoheitlichem Charakter gewährleistet ist. (VerfGH Berlin, a.a.O.) Die sachlich-inhaltliche Legitimation als Teil des demokratischen Prinzips verlangt, dass eine Bindung der hoheitlich handelnden Amtsträger an den Willen und die Weisungen einer der Volksvertretung oder dem Volk direkt verantwortlichen Spitze der Exekutive besteht. (BVerfGE 83, 60/ 73; 93, 37/ 66 ff.; BVerwGE 106, 64/74)

Diese Weisungsbeziehung zwischen den Amtswaltern und der demokratisch bestellten Spitze der Exekutive entsteht im Falle der Beleihung zunächst aus dem Vorgang der Beleihung selbst. Die sachlich-inhaltliche demokratische Legitimation folgt zunächst aus dem Institut und dem Vorgang der Beleihung. Die beliehenen Unternehmen werden aufgrund des Beleihungsgesetzes Träger öffentlicher Verwaltung; sie sind damit Verwaltungsbehörde im funktionalen Sinne und im Sinne des § 1 Abs. 4 VwVfG. (Wolff/Bachof/Stober, Verwaltungsrecht II, 5. Aufl. 1987, § 104 Rdnr. 10 m.w.N.) Damit unterliegen sie denselben Bindungen, die sich für die unmittelbare Staatsverwaltung aus dem verfassungsrechtlichen Demokratiegebot ergeben. Die Weisungsbefugnis kann sich deshalb nicht auf den einmaligen Akt der Beleihung beschränken, sondern muss aus dem Prinzip der demokratischen Kontrollierbarkeit und Verantwortung exekutivischen Handelns folgend fortbestehen. Wenn die Beliehenen staatliche Hoheitsgewalt ausüben, gebieten es die »Grundsätze demokratischer Legitimation, Verantwortlichkeit und Kontrolle, dass ihre Ausübung der Aufsicht eines - parlamentarisch verantwortlichen - Mitgliedes« der gewählten Regierung unterliegt, wobei die 
Aufsicht nicht nur als bloße Rechtsaufsicht, sondern als Fachaufsicht zu gestalten ist. Der Beliehene muss seine Aufgaben nach den Richtlinien und Weisungen des zuständigen demokratisch legitimierten Teils der Exekutive erfüllen. (StGH Bremen St 1/01 v. 15.1.2002, S. 22 f.)

Weil die funktionelle Privatisierung von Verwaltungsaufgaben nicht zur Entstehung kontrollfreier Räume öffentlicher Verwaltung führen darf (Spannowsky, DVBl. 1992, S. 1073/ 1073; Schoch, DVB1. 1994, S. 962/ 974 f.; Schuppert, DÖV 1998, S. 831 ff./ 833; Pitschas, DÖV 1998, S. 907 ff./ 910 ff.), gibt es eine verfassungsrechtliche Pflicht, dafür Sorge zu tragen, dass ausreichend und qualifiziertes Personal zur Verfügung steht, um die Kontroll- und Weisungsaufgaben effektiv wahrzunehmen. Maßstab für den Staatsgerichtshof Bremen ist die »rechtsstaatlich-demokratische Qualität der erbrachten öffentlichen Leistung « (StGH Bremen St 1/01 v. 15.1.2002, S. 23), die von der substanziellen Qualität der Leistung zu unterscheiden ist. Die geforderte rechtsstaatlich-demokratische Qualität mündet in die Verpflichtung, eine ausreichende behördliche Kontrolle der funktionell privatisierten Staatsaufgaben sicher zu stellen.

\section{PPP und die Vermischung der Interessen}

Sinn der PPP ist es vordergründig, privates Kapital für staatliche Aufgaben zu mobilisieren. Verbunden mit dieser Einbeziehung privaten Kapitals in Unternehmungen oder Einrichtungen mit öffentlichen Aufgaben ist jedoch zumindest immer eine Teil-Umstellung der Logik der Aufgabenerfüllung. Denn Private dürften sich in der Regel nicht altruistisch an einem PPP-Projekt beteiligen, sondern die Erwartung haben, die übliche Kapitalrendite auch bei einer Investition in öffentliche Unternehmen zu erwirtschaften. Die Logik der öffentlichen Aufgabenerfüllung ist jedoch um die dem Allgemeinwohl förderliche Erbringung allgemeiner Dienste und Infrastrukturleistungen zentriert, während die ökonomische Effizienz aus dieser Perspektive nachgeordnetes Ziel ist und sinnvollerweise sein muss. Aufgrund der unterschiedlichen Logiken des privaten und öffentlichen Sektors ist es wahrscheinlich, dass die Interessen der privaten Gesellschafter mit der staatlichen Gemeinwohlorientierung an der ein oder anderen Stelle kollidieren. Der Staatsgerichtshof Bremen beschreibt dieses Kollisionsproblem unter verfassungsrechtlich, normativen Gesichtspunkten folgendermaßen: »Wenn Informationsbegehren oder Weisungen der Behörde unter Hinweis auf das gesellschaftsrechtlich geschützte Interesse an der Geheimhaltung bestimmter Vorgänge oder auf entgegenstehende Gesellschaftsinteressen oder Abstimmungsergebnisse verweigert werden können, hängt die Erfüllung öffentlicher Aufgaben nicht von den Entscheidungen staatlicher Amtswalter, sondern von der Willensbildung in den Organen der Gesellschaft ab. Dies wäre mit dem Gebot demokratischer Legitimation der Erfüllung öffentlicher Aufgaben nicht vereinbar. Darüber hinaus entsteht bei beliehenen Unternehmen, insbesondere solchen mit gemeinschaftlicher öffentlicher und privater Beteiligung, die neben der Erfüllung von Verwaltungsaufgaben auch erwerbswirtschaftliche Ziele verfolgen, die Gefahr einer Vermischung privater und öffentlicher Handlungsziele, -maßstäbe und -motive. Der damit drohende Mangel an Transparenz des Unternehmenshandelns erschwert dessen Steuerung und Kontrolle durch die Ver- 
waltung und in der Folge auch die parlamentarische Kontrolle der Exekutive.« (StGH Bremen St 1/01 v. 15.1.2002, S. 24 f.) Der Staatsgerichtshof hat aus dieser Problembeschreibung gefolgert, dass die demokratische Rückbindung vertraglich abgesichert werden muss, allerdings offen gelassen, wie dies zu geschehen hat, insbesondere hat er auf die Festschreibung einer öffentlichen Mindestbeteiligung verzichtet.

Eine solche Mindestbeteiligung hat der Bundespräsident richtigerweise in seiner Begründung zur Verweigerung der Gegenzeichnung des Flugsicherungsprivatisierungsgesetzes mit Blick auf die geforderte Kontrolle und Einflussmöglichkeit der demokratisch legitimierten Organe verlangt: »Darüber hinaus verfügt der Bund, wenn er - wie vorgesehen - nur noch 25,1 \% der Anteile an der Flugsicherungsorganisation hält, gesellschaftsrechtlich nicht über die verfassungsrechtlich zwingenden Instrumente zur operativen Steuerung des Unternehmens. Eine Sperrminorität von 25,1\% vermittelt nur eine Vetoposition. So können zwar Satzungsänderungen verhindert werden, eine Einflussnahme auf die operative Geschäftsführung ist rechtlich aber nicht möglich.« (http://www.bundespraesident.de/dokumente/-,2.633675/\%20Pressemitteilung/dokument.htm) Im Falle der Flugsicherung ist zu bedenken, dass das GG eine Sonderregelung enthält. Da die Vermischung von Interessen und Umsteuerung der Logiken grundsätzlich ein Problem bei der Vermischung von privaten und öffentlichen Organisationen ist, kann die Überlegung verallgemeinert werden. Die Übertragung hoheitlicher Aufgaben verlangt im Falle der Übertragung an rein Private zwingend, die Beleihung so auszugestalten, dass der Private als Teil der Verwaltung agiert und den fachaufsichtlichen Weisungen und Kontrollen, die auch personell effektiv ausgestaltet werden müssen, unterliegt. Da im Falle der PPP Interessenskollisionen wahrscheinlich sind, die eine faktische Aufgabe der demokratischen Steuerung zur Folge haben können, ist es hier verfassungsrechtlich gefordert, dass der Staat sich Mehrheitsrechte vorbehält, damit die demokratisch-rechtsstaatliche Qualität der Ausübung hoheitlicher Aufgaben gesichert bleibt.

\section{5. Übertragung von Entscheidungsbefugnissen über die Hochschulen}

Eine besondere Form der privaten Beteiligung an öffentlichen Entscheidungen hat sich im Hochschulrecht mit der Etablierung unterschiedlicher Formen von Hochschulräten entwickelt. Mit den neuen Formen werden die Hochschulen wie Aktiengesellschaften organisiert - neben den »Vorstand « mit dem Universitätspräsidium tritt ein »Aufsichtsrat«, der in den Ländern unterschiedlich starke Kompetenzen hat. Während der Hochschulrat beispielsweise in Brandenburg wohl eher beratende Funktionen hat, besitzt der Hochschulrat nach dem »Hochschulfreiheitsgesetz« NRW starke Entscheidungsbefugnisse, die mit dem Demokratieprinzip und der Wissenschaftsfreiheit nicht $\mathrm{zu}$ vereinbaren sind.

Zu den wichtigen Entscheidungen, die der Hochschulrat in NRW treffen darf, gehören nach § 21 HFG die Beschlussfassung über den Hochschulentwicklungsplan und über sog. Zielvereinbarungen, die zwischen Hochschule und dem Ministerium geschlossen werden. Diese Zielvereinbarungen sollen ( $\$ 6$ HFG) über strategische Entwicklungsziele sowie konkrete Leistungsziele abgeschlossen werden. Entscheidend 
ist: Die Hochschulfinanzierung wird von der Erreichung dieser Ziele abhängig gemacht: »Insbesondere kann ein Teil des Landeszuschusses an die Hochschulen nach Maßgabe der Zielerreichung gewährt werden«. Mindestens die Hälfte der Mitglieder des Hochschulrates dürfen nicht der Hochschule angehören, er kann aber auch vollständig mit Externen besetzt werden. Das Rekrutierungsverfahren ist bemerkenswert. Eine Findungskommission, in der Senat und bestehender Hochschulrat jeweils zwei Vertreter, das Ministerium einen Vertreter mit zwei Stimmen entsendet, stellt den Hochschulrat zusammen. Der Hochschulrat rekrutiert sich also z.T. selbst. Der Senat der Hochschule muss der Auswahl mit Mehrheit zustimmen.

Die Selbstverwaltung der Hochschule wird gleichzeitig reduziert auf die Bestätigung der Auswahl des Hochschulrates und der Bestätigung der »Wahl« der Mitglieder des Präsidiums, also der Hochschulleitung, die im Hochschulrat stattfindet. Außerdem darf der Senat noch die Grundordnung der Universität erlassen und ändern, also darüber befinden, ob die Hochschule vollständig von Externen gesteuert wird, oder ob Mitglieder der Hochschule zur Hälfte im Hochschulrat vertreten sein dürfen. Wenn der Hochschulrat paritätisch besetzt ist, entscheidet der Vorsitzende bei Stimmengleichheit. Der Vorsitzende muss selbstverständlich ein Externer sein. Zum Hochschulentwicklungsplan, den Zielvereinbarungen usw. darf der Senat nur noch Stellungnahmen abgeben.

Dieses Neuorganisation der Hochschule muss an der Wissenschaftsfreiheit Art. 5 Abs. 3 GG gemessen werden. Diese nordrheinwestfälischen Regelungen sind nicht verfassungskonform. Umfassend ausgelegt wurde Art. 5 Abs. 3 GG vom BVerfG in zwei Urteilen: dem älteren Hochschulurteil aus dem Jahr 1973, bei dem es um die Drittelparität in Niedersachsen ging (BVerfGE 35, 79) und dem jüngeren Urteil aus 2004 zum Hochschulgesetz in Brandenburg, bei dem u.a. auch ein Hochschulrat auf seine Verfassungskonformität geprüft wurde (BVerfGE 111, 333).

Im Urteil aus 1973 stellte das BVerfG grundsätzlich fest, dass die Wissenschaftsfreiheit durch die Art der Hochschulorganisation beeinträchtigt werden kann, nämlich dann, wenn die anderen Statusgruppen in den Selbstverwaltungsgremien die Mehrheit haben. Das Gericht hat Art. 5 Abs. 3 GG sehr weit ausgelegt und damit die damaligen Reformbestrebungen beschränkt. An der grundsätzlichen Aussage hat das Gericht allerdings auch im Jahr 2004 bei der Beurteilung der brandenburgischen Hochschulorganisation festgehalten: »Der Staat muss danach für funktionsfähige Institutionen eines freien Wissenschaftsbetriebs sorgen und durch geeignete organisatorische Maßnahmen sicherstellen, dass das individuelle Grundrecht der freien wissenschaftlichen Betätigung so weit unangetastet bleibt, wie das unter Berücksichtigung der anderen legitimen Aufgaben der Wissenschaftseinrichtungen und der Grundrechte der verschiedenen Beteiligten möglich ist. ... Wissenschaft ist ein grundsätzlich von Fremdbestimmung freier Bereich autonomer Verantwortung. Dem Freiheitsrecht liegt auch der Gedanke zu Grunde, dass eine von gesellschaftlichen Nützlichkeits- und politischen Zweckmäßigkeitsvorstellungen freie Wissenschaft Staat und Gesellschaft im Ergebnis am besten dient « (BVerfGE 111, 333/354).

Die organisationspolitische Sicherung der Forschungs- und Wissenschaftsfreiheit, erfordert es, folgerte das BVerfG 1973, dass Hochschullehrer in Gremien, in denen 
Entscheidungen über Fragen der Wissenschaft und Forschung fallen, mit Mehrheit vertreten sind. Den Hochschullehrern müsse eine herausgehobene Stellung zukommen, weil ihnen die Pflege von Forschung und Lehre vornehmlich anvertraut ist (BVerfGE 35, 79/125).

In der neueren Entscheidung wird der Prüfungsmaßstab enger geschnürt: »Zur Klärung der Frage, ob eine Regelung Strukturen schafft, die sich gefährdend auswirken können, ist das hochschulorganisatorische Gesamtgefüge mit seinen unterschiedlichen Einfluss- und Kontrollmöglichkeiten in den Blick zu nehmen. $\mathrm{Zu}$ berücksichtigen ist dabei auch der Grad der Bedeutung der jeweils zu treffenden Entscheidung für die freie wissenschaftliche Betätigung und Aufgabenerfüllung. Da sich die meisten hochschulorganisatorischen Entscheidungen, auch wenn sie den Kernbereich wissenschaftlicher Betätigung nicht unmittelbar berühren, aufgrund der Angewiesenheit der wissenschaftlich Tätigen auf den öffentlich bereitgestellten und organisierten Wissenschaftsbetrieb mittelbar auf die wissenschaftliche Betätigung auswirken können, reicht eine nur hypothetische Gefährdung nicht aus« BVerfGE 111, 333/355). Dies wird ergänzt durch den Hinweis auf den weiten Einschätzungsspielraum des Gesetzgebers und ein mögliches Experimentierfeld, weshalb die alte Form der Selbstverwaltung nicht die einzige Form sei, in der Hochschule organisiert werden könne.

Im Ergebnis seien mit dem brandenburgischen Hochschulrat noch keine Strukturen geschaffen worden, die in die Autonomie der Wissenschaft eingriffen. Das lässt sich mit der eher beratenden Funktion des Hochschulrates begründen, die Entscheidungsrechte bei den Organen der Selbstverwaltung und den staatlichen, demokratisch legitimierten Institutionen belässt.

Der Hochschulrat in Brandenburg entscheidet nicht über die Hochschulentwicklungsplanung und die Zielvereinbarungen, sondern er berät nur. »Seine Aufgaben ( 63 Abs. 2 BbgHG) sind überwiegend beratender und empfehlender Natur.« Weiter führt das Gericht aus: »Das Vorschlagsrecht des Landeshochschulrats für die Wahl der Hochschulleitung ( $\$ 63$ Abs. 2 Nr. 4 BbgHG) verletzt nicht Art. 5 Abs. 3 Satz 1 GG. Die Organisation eines grundrechtlichen Freiheitsbereichs mittels Einschaltung unabhängiger Gremien, um die Organisation des Freiheitsbereichs zwar staatsfrei, aber unter öffentlicher Kontrolle zu halten, ist verfassungsrechtlich unbedenklich. Gerade im Bereich der Wissenschaftsfreiheit kann die Einschränkung des herkömmlichen hierarchisch geprägten Kontrollinstrumentariums dem Grundrecht dienen.«

Anderswo, wie beispielsweise in NRW, sind die Kompetenzen des Hochschulrates deutlich weitgehender. Er berät nicht nur, sondern übernimmt Entscheidungsbefugnisse, welche die Wissenschaftsfreiheit unmittelbar betreffen. Im HFG-NRW finden sich etwa folgende Kompetenzen:

$\S 15$ I Nr. 2: Der HR bestimmt die Anzahl der nicht hauptberuflichen Mitglieder des Präsidiums.

$\S 16$ III: Das Präsidium ist gegenüber dem HR rechenschaftspflichtig in Bezug auf die Erfüllung der Aufgaben der Hochschule.

$\S 17$ I: Die hauptberuflichem Mitglieder des Präsidiums werden vom HR gewählt

$\S 17$ II: Die nicht hauptberuflichen Vizepräsidenten werden ebenfalls vom HR gewählt. 
$\S 17$ III: Bestätigt der Senat die beiden Wahlen nicht, kann der HR die Bestätigung mit qualifizierter Mehrheit ersetzen.

§ 17 IV: Der HR kann mit 2/3 Mehrheit einzelne Präsidiumsmitglieder abwählen.

$\S 18$ III: Der HR bestellt den Präsidenten.

$\S 21$ I Nr. 2: Der HR muss dem Hochschulentwicklungsplan und den Zielvereinbarungen zustimmen.

§ 21 I Nr. 3: Zustimmung zum Wirtschaftsplan und zur unternehmerischen Tätigkeit der Hochschule

§ 33 II: Der HR ist oberste Dienstbehörde.

Ein in dieser Weise installierter Hochschulrat ersetzt demokratisch legitimierte Entscheidungen durch solche einer sich selbst rekrutierenden Oligarchie, die sich letztlich aus der Wirtschaft rekrutiert. Staatliche Verantwortung wird damit auf Private übertragen, die damit in verfassungswidriger Weise die Entwicklung von Wissenschaft und Forschung mitsteuern und auf private Interessen verpflichten können. Und genau dies war das Anliegen bei der Neustrukturierung der Hochschulen. Die Entmachtung der Selbstverwaltung wird bereits am Beispiel der Universität Siegen offenkundig.. Dort wählte der Hochschulrat ohne Abstimmung mit dem Senat einen neuen Präsidenten. Der Senat, der mit der Wiederwahl des bisherigen Rektors gerechnet hatte, lehnte diesen mit der Mehrheit der Stimmen ab. Dennoch kann sich der Hochschulrat mit einer Zweidrittelmehrheit über dieses ablehnende Votum hinwegsetzen. Ob es dazu kommt, ist fraglich. Der vom Hochschulrat auserkorene Kandidat hat allerdings schon angekündigt, dass er seine Bewerbung nicht zurückziehen wird.

\section{Materielle Privatisierung und die am Allgemeinwohl orientierte Reproduktion}

\section{Gewährleistung der Reproduktion einer Marktgesellschaft}

Die Reproduktion einer marktvermittelten und wettbewerbsorientierten Wirtschaft ist auf staatliche Vorleistungen angewiesen, die nicht marktfähig sind oder privatwirtschaftlich nicht zur Verfügung gestellt werden. Die Sicherung der Reproduktionsbedingungen ist auch »in einer grundsätzlich marktwirtschaftlich geordneten Wirtschaft eine legitime Aufgabe staatlicher Wirtschaftspolitik, « formuliert das BVerfG, »die von der Verantwortung des Staates für den ungestörten Ablauf des wirtschaftlichen Geschehens im ganzen ausgeht.« Und das Gericht konkretisiert diese Verantwortung so: »Sie umfasst - als Strukturpolitik - auch die Planung und Durchführung von Maßnahmen, die den im Gefolge der technischen Entwicklung und der weltwirtschaftlichen Verflechtung ständig auftretenden Wandlungen ... Rechnung tragen.« (BVerfGE 30, 292/ 311 f.) Diese Verantwortung für die gesamtwirtschaftliche Reproduktion ist ebenso Kennzeichen moderner Staatlichkeit in einer kapitalistischen Gesellschaft wie das staatliche Gewaltmonopol.

$\mathrm{Zu}$ den Bereichen, die den ungestörten Ablauf des wirtschaftlichen Geschehens im Ganzen, die Reproduktion einer Marktökonomie sichern, gehören allgemeine Dienstleistungen und eine Infrastrukturpolitik, die sicherstellt, dass die für eine moderne Ökonomie notwendigen Netze und Strukturen vorhanden sind. In diesen Bereich ge- 
hört die Vorsorgeverwaltung, also die Bereitstellung von öffentlichen Einrichtungen für die Allgemeinheit wie Verkehrs- und Beförderungseinrichtungen, die Gas-, Wasser-, und Elektrizitätsversorgung (Ewald, Privatisierung staatlicher Aufgaben, S. 63) oder die Sicherung moderner Kommunikationsnetze. Dazu gehört die Sicherung der Gesundheitsversorgung ebenso wie die Sicherung eines für moderne Ökonomien ausreichenden Bildungsniveaus.

Die staatliche Funktion, die Reproduktionsbedingungen auch in einer Marktgesellschaft zu garantieren, sagt nicht, in welcher Form diese Sicherung erfolgen muss. Abgegrenzt werden auf diese Weise nur die öffentlichen Aufgaben, für die der Staat Verantwortung übernehmen muss oder historisch übernommen hat, von anderen, privaten Aufgaben. Staatliche Verantwortung besteht weiter in den Fällen der formellen oder funktionellen Privatisierung, auch wenn dies nicht selten verkannt wird. Gesetzlich wird diese staatliche Verantwortung für Infrastruktur und allgemeine Dienstleistungen auch in einigen Bereichen der materiellen Privatisierung normiert - man spricht in diesen Fällen von der Gewährleistungsverantwortung. Die Frage, inwieweit diese Verantwortung mit der materiellen Privatisierung vereinbar ist, kann nur anhand der verfassungsrechtlichen Normierungen zu den Bedingungen bundesrepublikanischer Staatlichkeit beantwortet werden.

\section{Verfassungsrechtliche Gewährleistungspflichten}

Verfassungsrechtliche Gewährleistungspflichten des Staates für allgemeine, öffentliche Dienste oder die Infrastruktur finden wir für den Bereich der Eisenbahnnetze, der Telekommunikation und Post sowie der Bildung.

Für die Eisenbahnnetze formuliert das GG explizit, dass der Bund eine angemessene Infrastruktur an Schienenwegen und die Versorgung mit Verkehrsangeboten sicherstellen muss. Gemäß Art. 87 e Abs. 4 GG gewährleistet der Bund, dass dem Wohl der Allgemeinheit, insbesondere den Verkehrsbedürfnissen, beim Ausbau und Erhalt des Schienennetzes der Eisenbahnen des Bundes sowie bei deren Verkehrsangeboten auf diesem Schienennetz Rechnung getragen wird.

Wie dem Wohl der Allgemeinheit Rechnung zu tragen ist, wird mit Blick auf die Organisationsform der Eisenbahn in Art. 87 e Abs. 3 GG geregelt, wonach die Wirtschaftsunternehmen zwar in privat-rechtlicher Form organisiert werden sollen. Soweit die Tätigkeit des Wirtschaftsunternehmens den Bau, die Unterhaltung und das Betreiben von Schienenwegen umfasst, muss dieses Unternehmen aber mehrheitlich im Eigentum des Bundes verbleiben. Der Wortlaut der Vorschrift ist eindeutig, die Veräußerung der Schienennetze ist verfassungsrechtlich unzulässig, d.h. eine volle materielle Privatisierung ist ausgeschlossen.

In Art. 87 e GG ist die Frage nach den Eigentumsverhältnissen explizit als Frage nach der öffentlichen Verantwortung bei privater Wirtschaftsform gestellt und in Beziehung gesetzt zum öffentlichen Mit-Eigentum an den privatrechtlich organisierten Unternehmen mit öffentlichen Aufgaben. Es wird explizit die Beziehung zwischen den Interessen des Allgemeinwohls und der staatlichen Verantwortung, die durch entsprechende Eigentumsverhältnisse gesichert werden, hergestellt. 
Für die Post und Telekommunikation normiert Art. 87 f. GG ebenfalls eine Gewährleistungspflicht des Staates für das Allgemeinwohl, das ebenso wie bei der Eisenbahn über eine angemessene Versorgung der Bevölkerung bestimmt wird. Im Bereich des Postwesens und der Telekommunikation muss danach der Bund flächendeckend angemessene und ausreichende Dienstleistungen gewährleisten. Im Bereich der Post wird diese Gewährleistungspflicht ebenfalls an die öffentliche Eigentümerstellung gekoppelt. Indem Art. 143 b Abs. 2 S. 2 GG bestimmt, dass der Bund die Kapitalmehrheit an den Nachfolgeunternehmen der Bundespost fünf Jahre nach der formellen Privatisierung veräußern darf. Die Systematik beider Vorschriften deutet darauf hin, dass regelmäßig das öffentliche Eigentum, auch wenn dieses formell privatisiert ist, die geeignete Form ist, um der Gewährleistungspflicht zu genügen.

Im Bereich der Schulversorgung macht Art. 7 GG dieses Regel-Ausnahme-Verhältnis ebenfalls deutlich. Art. 7 Abs.1 GG normiert zunächst die staatliche Verantwortung für das Bildungswesen. Gemäß Art. 7 Abs. 4 GG wird dann das Recht zur Errichtung von privaten Schulen gewährleistet. Die Privatschulen werden aber als Ausnahmeerscheinung konzipiert, schon weil Satz 2 bestimmt, dass sie nur als Ersatz für öffentliche Schulen fungieren und deshalb der Genehmigung des Staates bedürfen. Das BVerfG hat die Bedeutung und Funktion des staatlichen Schulwesen als Ergebnis historischer Erfahrungen gedeutet und anerkannt: »Die Trennung des Schulwesens in staatliche und private Schulen und der Primat staatlicher Schulen mit umfassendem Aufsichtsrecht auch über alle genehmigungspflichtigen privaten Schulen findet sich bereits im Allgemeinen Landrecht für die Preußischen Staaten von 1794. Die Übernahme des Schulwesens als staatliche Aufgabe - mit dem Ziel, unter anderem durch die Einführung einer allgemeinen Schulpflicht möglichst allen Bürgern ein Mindestmaß an Bildung und Ausbildung zu vermitteln - beendete eine jahrhundertelange Vormachtstellung der Kirchen, die sich bis dahin auch in Form der geistlichen Schulaufsicht über die zunächst von den Städten zugelassenen ständischen Schulen und gegen die weitgehend verbotenen sogenannten Klipp- oder Winkelschulen nicht ständisch organisierter Privatlehrer behauptet hatte (BVerfGE 75, 40/ 56 f.). Diese Ausführungen erhellen den doppelten Zweck des staatlich organisierten Bildungswesens, der erstens darin besteht, eine allgemeine, gleichmäßige allen Bürgern zugängliche Bildung zu sichern und zweitens die Bildung von besonderen Interessen und einer Vermachtung freizuhalten. Dies gilt in gleicher Weise für den Hochschulbereich.

\section{Allgemeine verfassungsrechtliche Bestimmungen und die öffentliche Gewährleis- tung}

Die expliziten Gewährleistungspflichten werden verfassungsrechtlich erweitert durch Bestimmungen, welche auf die Organisation der öffentlichen Aufgaben ausstrahlen. Dazu gehören das Sozialstaatsprinzip in Verbindung mit dem Gleichheitssatz sowie staatliche Schutzpflichten, die sich aus den Grundrechten ergeben. Das Sozialstaatsprinzip ist zu verstehen als Verpflichtung des Staates, die Mindestvoraussetzungen für »ein menschenwürdiges Dasein« zu schaffen, was bedeutet, dass die »Fürsorge für Hilfsbedürftige zu den selbstverständlichen Pflichten eines Sozialstaates« gehört. 
(BVerfGE 40, 121/ 133; 35, 202/ 236) Zur Erfüllung der sozialen Verpflichtungen wird dem Gesetzgeber ein weiter Ermessensspielraum eingeräumt, der ihn aber nicht von der grundsätzlichen Pflicht, sozialen Ausgleich anzustreben, entbindet. »Das Wesentliche zur Verwirklichung des Sozialstaates aber kann nur der Gesetzgeber tun; er ist gewiss verfassungsrechtlich zu sozialer Aktivität, insbesondere dazu verpflichtet, sich um einen erträglichen Ausgleich der widerstreitenden Interessen und um die Herstellung erträglicher Lebensbedingungen für alle ... zu bemühen.« (BVerfGE 1, 97/ 104) Die sozialstaatlichen Pflichten wurden von der höchstrichterlichen Rechtsprechung dennoch weiter konkretisiert. So formulierte das BVerfG: »Darüber hinaus entnimmt die freiheitliche demokratische Grundordnung dem Gedanken der Würde und Freiheit des Menschen die Aufgabe, auch im Verhältnis der Bürger untereinander für Gerechtigkeit und Menschlichkeit zu sorgen. ... Sie sieht es aber als ihre Aufgabe an, wirkliche Ausbeutung, nämlich Ausnutzung der Arbeitskraft zu unwürdigen Bedingungen und unzureichendem Lohn zu unterbinden. Vorzüglich darum ist das Sozialstaatsprinzip zum Verfassungsgrundsatz erhoben worden; es soll schädliche Auswirkungen schrankenloser Freiheit verhindern und die Gleichheit fortschreitend bis zu dem vernünftigerweise zu fordernden Maße verwirklichen.« (BVerfGE 5, 85/ 205 f.) Der allgemeine Gleichheitssatz des Art. 3 Abs. 1 GG, der zunächst die formale, d.h. rechtliche Gleichbehandlung verfassungsrechtlich gewährleistet, wird über das Sozialstaatsprinzip material aufgeladen, in dem vom BVerfG formulierten Sinn, dass ein Fortschreiten zur Gleichheit in der Differenz verfassungsrechtlicher Auftrag an den Gesetzgeber ist. Vor diesem Hintergrund lässt sich das Sozialstaatsprinzip als Ausformung und Konkretisierung der Staatlichkeit verstehen, welche Voraussetzung der Reproduktion der marktförmig organisierten Wirtschaft ist.

Die soziale Fürsorge für Hilfsbedürftige wurde vom BVerwG konkretisiert zu einem Anspruch auf Teilhabe an der sozio-kulturellen Entwicklung. So ergibt sich aus dem Sozialstaat das Recht auf ein »soziokulturelles Existenzminimum, das den Leistungsberechtigten nicht nur das zum Lebensunterhalt Unerlässliche gewährt, sondern sie in die Lage versetzen soll, in der Umgebung von Nichthilfeempfängern ähnlich wie Personen mit geringem Einkommen leben zu können.«(BVerwGE 36, 258) Öffentliche Aufgaben, die für die ökonomische und gesellschaftliche Reproduktion erforderlich sind, betreffen aber, soweit sie als allgemeine Dienste oder Produktion von meritorischen Gütern in Erscheinung treten, oftmals gerade solche Bereiche, für welche die Teilhabe gegenwärtig zum soziokulturellen Existenzminimum gehört. Dann folgt, dass der Staat diese allgemeinen Dienste nicht zwingend selbst erbringen muss, jedoch auch hier eine Gewährleistungspflicht besteht, und zwar eine Gewährleistungspflicht, die beinhaltet, dass entsprechende meritorische Güter, Infrastrukturen oder allgemeine Dienste diskriminierungsfrei, allgemein verfügbar vorgehalten werden. Das BVerfG hat eine entsprechende staatliche Verpflichtung aus dem Sozialstaatsprinzip für das Gesundheitswesen abgeleitet: »Im Sozialstaat des Grundgesetzes ist es Staatsaufgabe, die Krankenhausversorgung der Bevölkerung zu sozial tragbaren Kosten sicherzustellen.« Die Aufgabe muss der Staat nicht vollständig selbst übernehmen, führt das Gericht weiter aus, ist aber verantwortlich im Sinne einer Gewährleistungspflicht, die hier als Pflicht zum Selbsteintritt im Falle von Ausfällen zu verstehen ist: »Diese Auf- 
gabe kann der Staat dadurch erfüllen, dass er unter Einbeziehung freier gemeinnütziger und kommunaler Träger ein bedarfsgerechtes Gesamtsystem leistungsfähiger Krankenhäuser unterhält und diese in der Weise finanziert, dass er die erforderlichen Investitionskosten übernimmt und die verbleibenden notwendigen Selbstkosten durch entsprechend festgesetzte Pflegesätze aufbringen lässt« (BVerfGE 52, 366/ 410).

Die sozialstaatliche Gewährleistungspflicht ist in bestimmten Fällen - wie beispielsweise dem Gesundheitssystem - zu ergänzen um die grundrechtlichen Schutzpflichten, die sich aus Art. 2 Abs. 2 GG für Leben und Gesundheit ergeben. Dem Staat ist es danach nicht nur verwehrt, ohne verfassungsrechtliche Rechtfertigung in Leben und Gesundheit der Bürger einzugreifen, er hat vielmehr eine Schutzpflicht, also die Verpflichtung, aktiv Leben und Gesundheit der Bürger zu schützen (BVerfGE 39, 1/ 42). Die staatliche Handlungspflicht, die sich aus dem Gesichtspunkt der grundrechtlichen Schutzpflichten ergibt, wird als Untermaßverbot (BVerfGE 96, 406/412) bezeichnet. Auch hier besteht regelmäßig ein Ermessen des Staates, wie er dieser Schutzpflicht nachkommt, allerdings verbietet die Schutzpflicht es, untätig zu bleiben, wenn die marktwirtschaftliche Organisation absehbar nicht in der Lage ist, für eine angemessene Versorgung, beispielsweise Gesundheits- oder Wasserversorgung, die ebenfalls eine mögliche Gefährdung des Schutzgutes des Art. 2 Abs. 2 GG bedeuten würde, sicher zu stellen. Das Untermaßverbot gebietet ein Mindestmaß an Vorkehrungen, um einen Ausfall privater Leistungserbringung auszuschließen - was regelmäßig nur sehr beschränkt möglich ist - oder staatlich aufzufangen. Das heißt, der Staat trägt gleichsam eine Ausfallverantwortung, der er beispielsweise beim durch einen Investitionsstau bei den Netzen verursachten Ausfall der Stromversorgung nachkommen muss.

\section{Demokratische Gestaltung}

Wenn Art. 20 Abs. 1 GG bestimmt, dass die Bundesrepublik Deutschland ein demokratischer und sozialer Bundesstaat ist, und diese Regelung durch die Ewigkeitsgarantie des Art. 79 Abs. 3 GG erfasst wird; wenn weiter Art. 28 Abs. 1 GG bestimmt, dass die verfassungsmäßige Ordnung in den Ländern den Grundsätzen des republikanischen, demokratischen und sozialen Rechtsstaates entsprechen muss und in den Ländern, Kreisen und Gemeinden das Volk eine Vertretung haben muss, die aus allgemeinen, unmittelbaren, freien, gleichen und geheimen Wahlen hervorgegangen ist, dann folgt, dass der demokratische Aufbau des Staates auf den unterschiedlichen Ebenen einen zentralen Stellenwert in der Verfassung einnimmt, der es verbietet das Demokratieprinzip auf die Legitimation von Staatsgewalt und Herrschaft zu reduzieren. Es beinhaltet notwendig auch einen Gestaltungsauftrag an die Politik, der im Wege der Selbstgesetzgebung sowie der Steuerung und Kontrolle der Exekutive wahrzunehmen ist. Die Volksvertretungen auf allen Ebenen des Gesamtstaates setzen gleichsam voraus, dass sie Funktionen der Repräsentation des Volkes in der Politik wahrnehmen. Das BVerfG hat klargestellt, dass die normative Konstituierung einer Institution durch die Verfassung es gebietet, dass diese Institution auch faktisch Funktionen hat und diese Funktionen übernehmen kann (BVerfGE 69, 1/ 21). Diese Funktion, die einer demokratischen Volksvertretung vorausgesetzt ist, lässt sich als politische Gestaltung 
fassen, d.h. sie ist berechtigt, kollektiv verbindliche Entscheidungen zu treffen, mit denen Einfluss genommen wird auf die Frage »Wie wollen wir leben«. Das heißt aber, dass den Volksvertretungen ein Gestaltungsspielraum eröffnet werden muss, in dem kollektiv verbindliche Entscheidungen getroffen werden können - und dieser Gestaltungsspielraum muss nicht nur normativ existieren, sondern auch faktisch.

Wenn wichtige Kompetenzen, die sich auf die Frage »wie wollen wir leben« auswirken, also dem Bereich der politischen Gestaltung zugehören, Privaten übertragen werden, verringert sich offensichtlich der Gestaltungsspielraum der Volksvertretungen. Nun kann die Volksvertretung in bestimmten Grenzen selbst entscheiden, welche Aufgaben sie weiter wahrnehmen will und welche sie übertragen will, allerdings muss wegen der hohen Bedeutung des Art. 20 GG zumindest ein Kernbereich an funktionalen Kompetenzen bei der Volksvertretung bleiben. Das heißt: Bei der Beurteilung der Verfassungskonformität von Privatisierungen ist nicht nur im Einzelfall darauf zu achten, dass bei der Ausübung von Staatsgewalt die Legitimation gewahrt wird, es ist auch ein Gesamtbild zu zeichnen und zu beurteilen, ob der jeweils zuständigen Volksvertretung ein ausreichender Gestaltungsspielraum bleibt, oder anders gesagt: ob mit der Privatisierung in Kernbereiche der demokratischen Entscheidungskompetenz eingegriffen wird. Die Grenze scheint im kommunalen Bereich teilweise mindestens tangiert zu werden, so dass das Vertrauen in die lokale Regierung schwindet und die Kommune ihre Bedeutung als »Schule der Demokratie« verliert (Schneidereit/ Weizsäcker, in: Weizsäcker/ Young/ Finger, Grenzen der Privatisierung, 286).

\section{Folgerungen}

So ist als Ergebnis festzuhalten, dass sich aus den grundrechtlichen Schutzpflichten sowie dem Sozialstaatsprinzip i.V.m. dem allgemeinen Gleichheitssatz eine Gewährleistungspflicht des Staates ergibt, die die Pflicht begründet, über öffentliche Einrichtungen eine allgemeine, gleichmäßige Versorgung der Bevölkerung mit allgemeinen Diensten sicher zu stellen. Solche - im Sinne einer existenziellen Versorgung - zu gewährleistenden Bereiche betreffen neben den in der Verfassung speziell geregelten wie Eisenbahn, Post und Telekommunikation auch die Wasser-, Gesundheits-, und die Energieversorgung der Haushalte oder die Abfallentsorgung. Gewährleistungspflicht bedeutet wie ausgeführt nicht, dass der Staat die Aufgaben zwingend selbst erfüllen muss, dass aber eine materielle Privatisierung ausgeschlossen ist.

Allerdings ist verfassungsrechtlich eine erhöhte Begründungspflicht für die Privatisierung öffentlicher, dem Allgemeinwohl dienender Aufgaben zu erbringen. Die Gewährleistungspflicht legt es, wie die aufgezeigten Regel-Ausnahme-Verhältnisse gezeigt haben, nahe, dass die öffentlichen Aufgaben, nicht materiell privatisiert werden. Für die Wasserbewirtschaftung hat das BVerfG betont, dass wegen des an ihr bestehenden hohen öffentlichen Interesses eine privatwirtschaftliche Aufgabenerfüllung nicht in Betracht kommt, und der staatliche Bewirtschaftungsgrundsatz der verfassungsrechtlich gebotenen Sozialpflichtigkeit des Eigentums entspricht. Es sei nicht vertretbar, formuliert das Gericht, die Nutzung des Wassers dem »freien Belieben des Einzelnen zu überlassen oder die Nutzung nur mehr durch den - für frühere Verhält- 
nisse ausreichenden - Rechtsgrundsatz der ,Gemeinverträglichkeit ${ }^{\star}$ zu begrenzen. Die Bewältigung einer derart umfassenden, dem Gemeinwohl dienenden Aufgabe gehört zu den typischen Angelegenheiten des öffentlichen Rechts, die mit den Mitteln des Privatrechts kaum erfüllt werden können.«(BVerfGE 58, 300/ 344)

Für andere Bereiche beinhaltet die Gewährleistungspflicht, dass

- Strukturen geschaffen werden, die eine Regulierung und Kontrolle der privaten Aufgabenerfüllung erlaubt,

- erwiesene oder absehbare Qualitätsverluste oder Versorgungsprobleme der Privatisierung der öffentlichen Aufgabe entgegenstehen,

- sichergestellt ist, dass im Falle eines Ausfalls der privaten Anbieter, die öffentlichen Einrichtungen technisch, organisatorisch und personell in der Lage sind, die ausgefallene Aufgabenerfüllung zu übernehmen.

Eine Privatisierung der Streckennetze der Bahn ist verfassungsrechtlich unzulässig, was auch für eine schleichende Privatisierung durch die Hintertür gilt.

Eine Privatisierung der Wasserversorgung ist verfassungsrechtlich unzulässig. Das gleiche gilt für die Privatisierung des Straßennetzes: Selbst unter der Voraussetzung, dass man Art. 90 GG ändern würde, spricht die weiter bestehende Gewährleistungsverantwortung gegen eine Privatisierung, weil eine ausreichende, mit dem Sozialstaatsprinzip und den Schutzpflichten vereinbare Verkehrsinfrastrukturversorgung absehbar nicht mehr gewährleistet wäre.

Es mehren sich die Zeichen, die für eine verfassungsrechtliche Pflicht zur öffentlichen Reorganisation der Energieversorgung der Haushalte sprechen, da die monopolisierte Energieversorgung und der monopolisierte Netzbetrieb offenbar zu Lasten der Infrastruktur mit inzwischen eingetretenen Ausfällen führt und zweitens eine gemeinverträgliche Preisgestaltung der Energieversorgung gefährdet erscheint.

Zusammengefasst: Alle Fragen, die in Richtung Abbau von Hemmnissen der Privatisierung gehen, arbeiten mit einer falschen Prämisse. Es steht die Reorganisation des Öffentlichen auch aus verfassungsrechtlichen Gründen an. 